\title{
Functional capacity-based rehabilitation of patients with chronic stable left ventricular heart failure \\ Youssy S. Gergius ${ }^{a}$, Nehal E. El-Sheshtawy ${ }^{b}$, Nadia H. El-Arousi ${ }^{b}$, Mahmoud M. Fathalla ${ }^{b}$, Mohamed A. Abdel Rahman ${ }^{c}$, Ahmed M. Gharib ${ }^{d}$
}

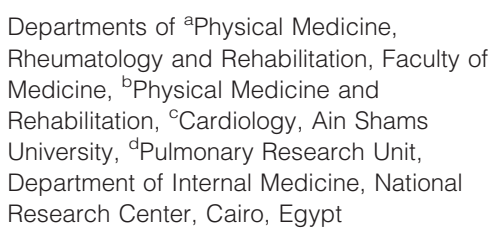

Departments of aphysical Medicine, Rheumatology and Rehabilitation, Faculty of Medicine, ${ }^{\text {bPhysical Medicine and }}$ Rehabilitation, ${ }^{\circ}$ Cardiology, Ain Shams University, dPulmonary Research Unit, Department of Internal Medicine, National Research Center, Cairo, Egypt

Correspondence to Youssy S. Gergius, BSc, MSc of Physical Medicine, Rheumatology and Rehabilitation, 27 Thabet Street, El Nozha El Gedida, Heliopolis, Cairo, 11843, Egypt. Tel: +20 1202565355

e-mail: uc_said@yahoo.com

Received 25 January 2018 Accepted 25 February 2018

Egyptian Rheumatology \& Rehabilitation 2018, 45:159-166

\begin{abstract}
Context
Heart failure (HF) is a common and costly condition. Reduced endurance is the main limiting factor of exercise capacity in HF patients. Cardiopulmonary exercise testing (CPX) is considered the most objective method to assess exercise capacity in HF patients.
\end{abstract}

Aim

To study the degree of improvement among patients with chronic stable left ventricular HF with low and average functional capacity after functional capacity-based rehabilitation program.

\section{Settings and design}

Rehabilitation was done at department of cardiology, department of physical medicine and rehabilitation, Ain Shams university. CPX was done at the National institute of research.

\section{Patients and methods}

A total of 40 patients with chronic heart failure were randomized to either a control (received their medical treatment with no specific rehabilitation program) or a rehabilitation group. Symptom-limited CPX was performed at baseline and at discharge from the program. Rehabilitation group was further divided according to their functional capacity measures obtained from CPX into group 1 and group 2 . Minnesota Living with Heart Failure Questionnaire was obtained from all participants. Group 1 received electric muscle stimulation (EMS) of both lower limbs 5 days/week for 5 weeks. Group 2 received a conventional aerobic rehabilitation program 2 or 3 times/week for $\sim 40$ sessions. $\mathrm{VO}_{2}$ peak, $\mathrm{VO}_{2}-\mathrm{VT}$, $\mathrm{VE} / \mathrm{VCO}_{2}$, peak load, heart rate recovery, and Minnesota Living with Heart Failure Questionnaire values were compared before and after the treatment period.

Statistical analysis used

Statistical presentation and analysis of the present study was conducted using the mean, SD, Student's $t$-test, paired $t$-test, $\chi^{2}$, linear correlation coefficient, and analysis of variance tests by SPSS, version 17 .

Results

EMS produced significant improvement of functional capacity measures in addition to quality of life. It was comparable to the aerobic rehabilitation in group 2. Both rehabilitation protocols caused significant improvement when compared with the control group.

\section{Conclusion}

Functional capacity and quality of life were improved after either EMS or aerobic rehabilitation protocol when applied to selected patients with chronic heart failure when compared with control patients who did not receive any rehabilitation program.

Keywords:

aerobic training, chronic heart failure, electromyostimulation, $\mathrm{VE} / \mathrm{VCO}_{2}$

Egypt Rheumatol Rehabil 45:159-166

(C) 2018 Egyptian Society for Rheumatology and Rehabilitation

$1110-161 X$

\section{Introduction}

The syndrome of chronic heart failure (CHF) has become one of the most common cardiovascular disorders throughout the world, thus placing a heavy financial and social burden on public health funding [1].

Recent evidence examining the underlying pathophysiology of fatigue and dyspnea, the two main symptoms in heart failure (HF), points to structural and functional abnormalities in skeletal muscle besides central hemodynamics disturbance. In symptomatic patients with $\mathrm{CHF}$, maximal exercise capacity is often less than $50 \%$ of normal. Exercise intolerance is a widespread and serious problem in patients with $\mathrm{CHF}[2]$. 
Cardiopulmonary exercise testing (CPX) proved to be the most accurate way for quantification of cardiorespiratory fitness, grading of the etiology and severity of impairment, and also an objective assessment of the response to intervention $[3,4]$.

The American Heart Association HF guidelines recommend exercise training for all stable outpatients with $\mathrm{HF}$ and a reducedleftventricular ejection fraction $[5,6]$.

However, for a variety of reasons, not all patients with $\mathrm{HF}$ are able to participate in traditional exercise, as patients with $\mathrm{CHF}$ tend to abandon physical training owing to the discomfort related to dyspnea and some even cannot undergo physical training owing to locomotor or central nervous system disorders $[7,8]$.

Neuromuscular electrical stimulation (NMES) of the lower limb muscles may be an alternative to physical training in patients with CHF [9]. NMES has been consistently shown to elicit positive skeletal muscle adaptations at an appropriate stimulus in patients unable to participate in traditional aerobic and/or resistance training programs [10].

Our aim is to study the degree of improvement in patients with chronic stable left ventricular $\mathrm{HF}$ with low and average functional capacity after functional capacity-based rehabilitation program.

\section{Patients and methods}

This study was conducted on 40 participants with chronic stable left-sided heart failure (LHF) with ejection fraction up to $40 \%$. Participants were recruited from the Cardiology Department and the Outpatient Clinic of Ain Shams University Hospitals. Eligible participants were women and men more than 20 years of age, who had well-compensated LHF at the time of entry into the study, with a diagnosis of HF of more than 3 months in duration, and with a stable medication regimen for at least 2 months. The study was approved by Faculty of Medicine, Ain Shams University Ethics Committee, and consent was obtained from all participants. Patients were excluded for the following conditions: unstable CHF; dyspnea at rest; myocardial infarction less than 3 months in duration before the study; uncontrolled arrhythmia; unstable angina pectoris as documented by anginal pain uncontrollable with medications and ST or T-wave elevation or depression at rest; uncontrolled hypertension or diabetes; obstructive valvular disease; congenital heart disease; cardiac transplantation; severe pulmonary hypertension or other severe pulmonary disease; history of hospitalization for intravenous treatment of $\mathrm{HF}$ within at least 3 months of inclusion; patients with coexisting neurological, orthopedic, peripheral vascular diseases, or any intercurrent infective or malignant disease that would interfere with the exercise training program; and participants in a specific exercise program before inclusion in the study.

\section{Patient assessment}

All patients who met the inclusion criteria underwent the following at baseline: full medical history taking, thorough clinical examination, and functional capacity assessment according to New York Heart Association classification [11] and the Goldman's specific activity scale [12]. Quality-of-life questionnaire using Minnesota Living with Heart Failure Questionnaire (MLHFQ) to measure the patient's perception of the effects of $\mathrm{HF}$ on his or her life [13]. Resting ECG is done to exclude any new ischemia or arrhythmias. Resting echocardiography by $2 \mathrm{D}$ Simpson method was used for confirmation of left ventricular ejection fraction, left ventricular dimensions, diastolic function, valvular diseases, and muscle condition. Cardiopulmonary treadmill exercise test was performed as an objective way of assessment of functional capacity [14]. It was done according to modified Naughton protocol [15] and analyzed using CareFusion Oxycon Pro, VIASYS/JAEGER LE200CE (Germany). We started with a warm-up phase with an initial treadmill speed and grade slope of $1.0 \mathrm{mph}$ and $0 \%$, respectively. The speed and/or grade were subsequently adjusted every 2 min to yield an approximate 1-MET increase per stage of exercise. Respiratory gas exchange data were determined continuously throughout the exercise test [14]. After the patients had achieved their peak workload, they were brought to $1 \mathrm{mph}$ and a $0 \%$ grade for an active recovery phase. This was maintained for $2 \mathrm{~min}$, after which they were seated for an additional $4 \mathrm{~min}$ of passive recovery. Maximum aerobic exercise capacity represented by peak oxygen uptake $\left(\mathrm{VO}_{2} \mathrm{p}\right)$ was defined as the highest oxygen uptake level achieved during the treadmill test [16]. Ventilatory threshold $\left(\mathrm{VO}_{2}-\mathrm{VT}\right)$ determined by the $\mathrm{V}$-slope method and $\mathrm{VE} / \mathrm{VCO}_{2}$ slope calculated over the whole exercise period were determined as powerful markers for disease severity and prognosis [17-19]. Heart rate recovery (HRR) was detected as a measure of the autonomic function. HRR was calculated as the difference between heart rate at peak exercise and at $1 \mathrm{~min}$ after exercise into active recovery [20]. Maximum workload achieved and causes of test termination were all noted. 


\section{Rehabilitation program}

Patients were randomized following baseline assessment into either control (10 patients) or rehabilitation group (30 patients). Rehabilitation group was further subdivided into group 1, including patients with low exercise capacity and poor prognosis represented by $\mathrm{VO}_{2}$-VT up to $11 \mathrm{ml} / \mathrm{min} / \mathrm{kg}$ and $\mathrm{VE} / \mathrm{VCO}_{2}$ slope of at least 35 , and group 2, including patients with average exercise capacity manifested as $\mathrm{VO}_{2}-\mathrm{VT}$ more than $11 \mathrm{ml} / \mathrm{min} / \mathrm{kg}$ and $/$ or $\mathrm{VE} / \mathrm{VCO}_{2}$ slope of less than 35 $[18,19]$. Control patients received their medical treatment and continued their previous level of physical activity at home with no special training program. Group 1 patients received a rehabilitation protocol in the form of electric myostimulation (EMS) of both lower limbs in addition to breathing exercises. Group 2 patients underwent a conventional aerobic training program.

\section{Electric myostimulation program}

The quadriceps and gastrocnemius muscles of both legs were electrically and simultaneously stimulated using adhesive electrodes and portable battery-powered fourchannel stimulator (Quadstar II; BioMedical Life Systems Inc., Vista, California, USA). On the quadriceps, two self-adhesive surface electrodes were positioned on the skin $\sim 5 \mathrm{~cm}$ below the inguinal fold and $3 \mathrm{~cm}$ above the upper patellar border. On the gastrocnemius, two electrodes were positioned $\sim 3 \mathrm{~cm}$ below the popliteal fossa and $5 \mathrm{~cm}$ above the Achilles tendon [21]. Blood pressure and HR were measured before and after each session. EMS was performed for $60 \mathrm{~min} /$ day, 5 days/week for a period of 5 weeks. Technical parameters of the stimulation were selected as follows: biphasic electric current with $10-\mathrm{Hz}$ frequency, in 'on-off' operation mode, with pulse duration of $200 \mu \mathrm{s}$. The time of ascent and descent of the current was $4 \mathrm{~s}$, and the time of contraction/relaxation was $20 \mathrm{~s}$. The intensity of stimulation was progressively increased to cause a visible contraction tolerable by the patient [19].

\section{Aerobic exercise protocol}

Each session is started and ended with a 10-min warmup and 5-min cool down period, respectively [22]. Exercise intensity was started at initial workload corresponding to $\sim 50-60 \%$ of maximal heart rate value obtained from the CPX. Duration was started at $15 \mathrm{~min}$ and was up-titrated gradually every week till the patient was able to perform 30 min continuously on the treadmill. Afterward, exercise intensity was increased gradually and progressively with target intensity of $\sim 85 \%$ of maximum heart rate. Sessions were held at a frequency of 2-3 sessions/week for an average of 40 sessions. Once patients demonstrated a tolerance of aerobic training levels (around 8-12 weeks for our patients), resistance training activities were added. Patients performed $10 \mathrm{~min}$ of light strength/ resistance training for both upper and lower limbs, focusing on the major muscle groups, using 1-2 sets of 10 repetitions/set [23].

\section{Follow-up}

Reassessment of all the recruited participants after the completion of the rehabilitation program was done by the following: clinical assessment, aerobic capacity (using CPX), and quality of life (using Minnesota living with HF questionnaire).

\section{The statistical analysis}

Statistical presentation and analysis of the present study was conducted, using the mean, SD, Student's $t$-test, paired $t$-test, $\chi^{2}$, linear correlation coefficient, and analysis of variance tests by SPSS, version 17 .

\section{Results}

A total of 46 patients met the inclusion criteria. Only 40 patients completed at least $90 \%$ of the rehabilitation program and the follow-up assessment. Control group had 10 patients, and groups 1 and 2 had 9 and 21 patients, respectively. The three groups were compared regarding age, BMI, EF, HF etiology, functional group, smoking, and history of hypertension and diabetes. There was a significant difference $(P<0.05)$ between the groups, where group 1 tended to be older, with higher BMI, and almost $90 \%$ of them had DM. Other variables did not show significant difference $(P>0.05)$. The control patients showed no statistically significant difference before and after treatment $(P>0.05)$ regarding $\mathrm{VO}_{2} \mathrm{p}$, ventilatory threshold $\left(\mathrm{VO}_{2}-\mathrm{VT}\right)$, maximum workload achieved, and HRR. However, there was a significant difference $(P<0.05)$ regarding $\mathrm{VE} / \mathrm{VCO}_{2}$ slope $(35.8 \pm 12.035-34.880 \pm 11.725)$ and MLHFQ (34.4 $\pm 16.695-32.1 \pm 16.224)$. EMS produced inferior improvements in $\mathrm{VO}_{2} \mathrm{p}$ when compared with the conventional aerobic training. However, it elicited superior improvements in $\mathrm{VO}_{2} \mathrm{p}$ compared with the control group, but still there was no significant difference in the change between group 1 and group 2 . Both EMS and aerobic training produced significant improvement in $\mathrm{VO}_{2}$-VT after rehabilitation; yet when comparing the degree of increase, there was a significant difference, where group 1 showed greater improvement than both the control and group 2. Both groups 1 and 2 showed significant difference after rehabilitation regarding $\mathrm{VE} / \mathrm{VCO}_{2}$ slope; however, group 1 with initial steeper slopes showed greater degree of improvement than control and group 2. Workload was 
Table 1 Comparison between the characteristics of all groups

\begin{tabular}{|c|c|c|c|c|c|c|c|c|c|c|}
\hline & & \multicolumn{3}{|c|}{ Control group (10) } & \multicolumn{3}{|c|}{ Group I (9) } & \multicolumn{3}{|c|}{ Group II (21) } \\
\hline & & $N$ & & $\%$ & $N$ & & $\%$ & $N$ & & $\%$ \\
\hline \multirow{2}{*}{ Functional class } & II & 8 & & 80.00 & 4 & & 44.44 & 13 & & 61.90 \\
\hline & III & 2 & & 20.00 & 5 & & 55.56 & 8 & & 38.10 \\
\hline \multirow[t]{3}{*}{ Smoking } & Non & 4 & & 40.00 & 3 & & 33.33 & 6 & & 28.57 \\
\hline & Smoker & 0 & & 0.00 & 0 & & 0.00 & 1 & & 4.76 \\
\hline & Ex-Smoker & 6 & & 60.00 & 6 & & 66.67 & 14 & & 66.67 \\
\hline \multirow[t]{2}{*}{ Etiology } & Ischemic & 9 & & 90.00 & 7 & & 77.78 & 18 & & 85.71 \\
\hline & Dilated & 1 & & 10.00 & 2 & & 22.22 & 3 & & 14.29 \\
\hline \multirow[t]{2}{*}{ DM } & No & 6 & & 60.00 & 1 & & 11.11 & 18 & & 85.71 \\
\hline & Yes & 4 & & 40.00 & 8 & & $88.89^{* a}$ & 3 & & 14.29 \\
\hline \multirow[t]{2}{*}{ HTN } & No & 6 & & 60.00 & 5 & & 55.56 & 14 & & 66.67 \\
\hline & Yes & 4 & & 40.00 & 4 & & 44.44 & 7 & & 33.33 \\
\hline \multirow[t]{2}{*}{ Age } & Range & 30 & - & 63 & 50 & - & 72 & 25 & - & 63 \\
\hline & Mean \pm SD & 52.200 & \pm & 9.852 & 59.556 & \pm & $7.601^{\star b}$ & 48.810 & \pm & 10.328 \\
\hline \multirow[t]{2}{*}{ EF } & Range & 25 & - & 38 & 20 & - & 39 & 22 & - & 40 \\
\hline & Mean \pm SD & 32.400 & \pm & 4.600 & 25.889 & \pm & $6.679^{\star C}$ & 31.238 & \pm & 5.495 \\
\hline \multirow[t]{2}{*}{ BMI } & Range & 23.5 & - & 36 & 23 & - & 38 & 23 & - & 31 \\
\hline & Mean \pm SD & 28.980 & \pm & 3.585 & 30.378 & \pm & $5.374^{\star b}$ & 26.262 & \pm & 2.322 \\
\hline
\end{tabular}

${ }^{\star} \mathrm{a}=P<0.05$, significant among the three groups. ${ }^{*} \mathrm{~b}=P<0.05$, significant between group 1 and 2 . ${ }^{\star} \mathrm{C}=P<0.05$, significant between control and group 1. DM=Diabetes Mellitus; HTN=Arterial Hypertension; EF=Ejection Fraction; BMI=Body mass index.

Table 2 Comparison between the control and rehabilitation patients regarding peak Vo2 pre and post rehabilitation

\begin{tabular}{|c|c|c|c|c|c|c|c|c|c|c|c|c|c|c|c|}
\hline \multirow{2}{*}{\multicolumn{2}{|c|}{ VO2 peak }} & \multicolumn{9}{|c|}{ Groups } & \multicolumn{2}{|c|}{ ANOVA } & \multicolumn{3}{|c|}{ TUKEY'S Test } \\
\hline & & \multicolumn{3}{|c|}{ Control } & \multicolumn{3}{|c|}{ Group I } & \multicolumn{3}{|c|}{ Group II } & \multirow{2}{*}{$\frac{F}{7.460}$} & \multirow{2}{*}{$\frac{P \text {-value }}{0.002^{\star}}$} & \multirow{2}{*}{$\frac{C \& I}{0.409}$} & \multirow{2}{*}{$\frac{\text { C\&II }}{0.073}$} & \multirow{2}{*}{$\frac{|\&| \mid}{0.002^{\star}}$} \\
\hline Pre & $\begin{array}{l}\text { Range } \\
\text { Mean } \pm S D\end{array}$ & $\begin{array}{c}6 \\
15.350\end{array}$ & $\begin{array}{l}- \\
\pm\end{array}$ & $\begin{array}{c}23 \\
5.467\end{array}$ & $\begin{array}{c}7 \\
12.589\end{array}$ & $\begin{array}{l}- \\
\pm\end{array}$ & $\begin{array}{c}19 \\
4.988\end{array}$ & $\begin{array}{c}13 \\
19.410\end{array}$ & $\begin{array}{l}- \\
\pm\end{array}$ & $\begin{array}{c}26.3 \\
4.087\end{array}$ & & & & & \\
\hline Post & $\begin{array}{l}\text { Range } \\
\text { Mean } \pm S D\end{array}$ & $\begin{array}{c}6.2 \\
15.670\end{array}$ & $\begin{array}{l}- \\
\pm\end{array}$ & $\begin{array}{c}24 \\
5.823\end{array}$ & $\begin{array}{c}8.5 \\
13.744\end{array}$ & $\begin{array}{l}- \\
\pm\end{array}$ & $\begin{array}{c}21 \\
5.089\end{array}$ & $\begin{array}{c}13 \\
21.738\end{array}$ & $\begin{array}{l}- \\
\pm\end{array}$ & $\begin{array}{c}33 \\
5.193\end{array}$ & 8.858 & $0.001^{*}$ & 0.714 & $0.014^{*}$ & $0.002^{*}$ \\
\hline \multicolumn{2}{|c|}{ Paired Differences } & -0.320 & \pm & 1.028 & -1.156 & \pm & 0.984 & -2.329 & \pm & 1.881 & 6.150 & $0.005^{\star}$ & 0.473 & $0.005^{*}$ & 0.150 \\
\hline \multicolumn{2}{|c|}{$\begin{array}{l}\text { Paired Samples } \\
\text { Test }\end{array}$} & \multicolumn{3}{|c|}{0.351} & \multicolumn{3}{|c|}{$0.008^{*}$} & \multicolumn{3}{|c|}{$<0.001^{*}$} & & & & & \\
\hline
\end{tabular}

Table 3 Comparison between the control and rehabilitation patients regarding Vo2-VT pre and post rehabilitation

\begin{tabular}{|c|c|c|c|c|c|c|c|c|c|c|c|c|c|c|c|}
\hline \multirow{2}{*}{\multicolumn{2}{|c|}{ VO2-VT }} & \multicolumn{9}{|c|}{ Groups } & \multicolumn{2}{|c|}{ ANOVA } & \multicolumn{3}{|c|}{ TUKEY'S Test } \\
\hline & & \multicolumn{3}{|c|}{ Control } & \multicolumn{3}{|c|}{ Group I } & \multicolumn{3}{|c|}{ Group II } & \multirow{2}{*}{$\begin{array}{c}F \\
19.177\end{array}$} & \multirow{2}{*}{$\frac{P \text {-value }}{<0.001^{*}}$} & \multirow{2}{*}{$\frac{\text { C\&I }}{0.117}$} & \multirow{2}{*}{$\frac{\text { C\&II }}{0.002^{*}}$} & \multirow{2}{*}{$\frac{I \& I I}{<0.001^{*}}$} \\
\hline Pre & $\begin{array}{l}\text { Range } \\
\text { Mean } \pm S D\end{array}$ & $\begin{array}{c}5.9 \\
11.540\end{array}$ & $\begin{array}{l}- \\
\pm\end{array}$ & $\begin{array}{c}17 \\
3.260\end{array}$ & $\begin{array}{c}6 \\
8.778\end{array}$ & $\begin{array}{l}- \\
\pm\end{array}$ & $\begin{array}{c}11 \\
2.279\end{array}$ & $\begin{array}{c}11.4 \\
15.686\end{array}$ & $\begin{array}{l}- \\
\pm\end{array}$ & $\begin{array}{c}21 \\
3.033\end{array}$ & & & & & \\
\hline Post & $\begin{array}{l}\text { Range } \\
\text { Mean } \pm S D\end{array}$ & $\begin{array}{c}5.3 \\
11.670\end{array}$ & $\begin{array}{l}- \\
\pm\end{array}$ & $\begin{array}{c}17 \\
3.590\end{array}$ & $\begin{array}{c}7.3 \\
10.756\end{array}$ & $\begin{array}{l}- \\
\pm\end{array}$ & $\begin{array}{c}15 \\
2.978\end{array}$ & $\begin{array}{c}11 \\
16.762\end{array}$ & $\begin{array}{l}- \\
\pm\end{array}$ & $\begin{array}{c}22 \\
3.279\end{array}$ & 14.196 & $<0.001^{*}$ & 0.819 & $0.001^{*}$ & $<0.001^{*}$ \\
\hline \multicolumn{2}{|c|}{$\begin{array}{l}\text { Paired } \\
\text { Differences }\end{array}$} & -0.130 & \pm & 0.826 & -1.978 & \pm & 0.992 & -1.076 & \pm & 0.680 & 12.906 & $<0.001^{*}$ & $<0.001^{*}$ & $0.010^{*}$ & $0.019^{*}$ \\
\hline \multicolumn{2}{|c|}{$\begin{array}{l}\text { Paired Samples } \\
\text { Test }\end{array}$} & \multicolumn{3}{|c|}{0.631} & \multicolumn{3}{|c|}{$<0.001^{*}$} & \multicolumn{3}{|c|}{$<0.001^{*}$} & & & & & \\
\hline
\end{tabular}

improved after rehabilitation in both groups 1 and 2 , with no significant difference in the degree of increase in WL between EMS and aerobic training. HRR showed only significant improvement after aerobic training in group 2. Quality of life manifested by MLHFQwas improved in all three groups after treatment, with the magnitude of the improvement being greatest in group 1 (Tables 1-7).

\section{Discussion}

Reduced exercise capacity is the cardinal symptom of CHF [24]. Almost 20\% of stable ambulatory patients with $\mathrm{HF}$ are affected by muscle wasting, with serious clinical implications, evidenced by worse exercise capacity [25]. CPX is considered a valuable tool in identifying patients who respond and those who do not to a given therapeutic intervention, as reflected by the change in CPX [26]. Regular aerobic physical activity is recommended to improve functional capacity and reduce morbidity and mortality in patients with $\mathrm{HF}$ [27]. NMES has shown to elicit positive skeletal muscle adaptations in some patients [10].

The value of $\mathrm{VO}_{2}-\mathrm{VT}(11 \mathrm{ml} / \mathrm{min} / \mathrm{kg})$ [28], along with the enhanced ventilatory response to exercise manifested with a steeper $\mathrm{VE} / \mathrm{VCO}_{2}$ slope of at least 35 , a 
Table 4 Comparison between the control and rehabilitation patients regarding VE/VCO2 slope pre and post rehabilitation

\begin{tabular}{|c|c|c|c|c|c|c|c|c|c|c|c|c|c|c|c|}
\hline \multirow{2}{*}{\multicolumn{2}{|c|}{ VE/VCO2 }} & \multicolumn{9}{|c|}{ Groups } & \multicolumn{2}{|c|}{ ANOVA } & \multicolumn{3}{|c|}{ TUKEY'S Test } \\
\hline & & \multicolumn{3}{|c|}{ Control } & \multicolumn{3}{|c|}{ Group I } & \multicolumn{3}{|c|}{ Group II } & \multirow{2}{*}{$\begin{array}{c}F \\
10.685\end{array}$} & \multirow{2}{*}{$\begin{array}{c}P \text {-value } \\
<0.001^{*}\end{array}$} & \multirow{2}{*}{$\begin{array}{c}\text { C\&I } \\
0.321\end{array}$} & \multirow{2}{*}{$\frac{\text { C\&II }}{0.022^{*}}$} & \multirow{2}{*}{$\frac{|\& \||}{<0.001^{*}}$} \\
\hline Pre & $\begin{array}{l}\text { Range } \\
\text { Mean } \pm S D\end{array}$ & $\begin{array}{c}25 \\
35.800\end{array}$ & $\begin{array}{l}- \\
\pm\end{array}$ & $\begin{array}{c}65 \\
12.035\end{array}$ & $\begin{array}{c}36 \\
40.889\end{array}$ & $\begin{array}{l}- \\
\pm\end{array}$ & $\begin{array}{c}51 \\
5.159\end{array}$ & $\begin{array}{c}16 \\
27.690\end{array}$ & $\begin{array}{l}- \\
\pm\end{array}$ & $\begin{array}{c}34 \\
5.510\end{array}$ & & & & & \\
\hline Post & $\begin{array}{l}\text { Range } \\
\text { Mean } \pm \mathrm{SD}\end{array}$ & $\begin{array}{c}24 \\
34.880\end{array}$ & $\begin{array}{l}- \\
\pm\end{array}$ & $\begin{array}{c}63 \\
11.725\end{array}$ & $\begin{array}{c}34 \\
37.444\end{array}$ & $\begin{array}{l}- \\
\pm\end{array}$ & $\begin{array}{c}44 \\
3.321\end{array}$ & $\begin{array}{c}16.5 \\
26.952\end{array}$ & \pm & $\begin{array}{c}35 \\
5.538\end{array}$ & 8.252 & $0.001^{*}$ & 0.723 & $0.019^{*}$ & $0.002^{*}$ \\
\hline \multicolumn{2}{|c|}{$\begin{array}{l}\text { Paired } \\
\text { Differences }\end{array}$} & 0.920 & \pm & 0.976 & 3.444 & \pm & 2.351 & 0.738 & \pm & 1.437 & 9.656 & $<0.001^{*}$ & $0.004^{*}$ & 0.953 & $<0.001^{*}$ \\
\hline \multicolumn{2}{|c|}{$\begin{array}{l}\text { Paired Samples } \\
\text { Test }\end{array}$} & \multicolumn{3}{|c|}{$0.015^{*}$} & \multicolumn{3}{|c|}{$0.002^{*}$} & \multicolumn{3}{|c|}{$0.029^{*}$} & & & & & \\
\hline
\end{tabular}

Table 5 Comparison between the control and rehabilitation patients regarding peak workload (WL) pre and post rehabilitation

\begin{tabular}{|c|c|c|c|c|c|c|c|c|c|c|c|c|c|c|c|}
\hline \multirow{2}{*}{\multicolumn{2}{|c|}{$\mathrm{WL}$}} & \multicolumn{9}{|c|}{ Groups } & \multicolumn{2}{|c|}{ ANOVA } & \multicolumn{3}{|c|}{ TUKEY'S Test } \\
\hline & & \multicolumn{3}{|c|}{ Control } & \multicolumn{3}{|c|}{ Group I } & \multicolumn{3}{|c|}{ Group II } & \multirow{2}{*}{$\frac{F}{5.699}$} & \multirow{2}{*}{$\frac{P \text {-value }}{0.007^{\star}}$} & \multirow{2}{*}{$\frac{C \& I}{0.940}$} & \multirow{2}{*}{$\frac{\text { C\&II }}{0.037^{*}}$} & \multirow{2}{*}{$\frac{|\&| \mid}{0.018^{*}}$} \\
\hline Pre & $\begin{array}{l}\text { Range } \\
\text { Mean } \pm S D\end{array}$ & $\begin{array}{c}30 \\
79.800\end{array}$ & $\begin{array}{l}- \\
\pm\end{array}$ & $\begin{array}{c}110 \\
26.541\end{array}$ & $\begin{array}{c}30 \\
75.111\end{array}$ & $\begin{array}{l}- \\
\pm\end{array}$ & $\begin{array}{c}122 \\
40.680\end{array}$ & $\begin{array}{c}70 \\
109.810\end{array}$ & $\begin{array}{l}- \\
\pm\end{array}$ & $\begin{array}{c}195 \\
26.832\end{array}$ & & & & & \\
\hline Post & $\begin{array}{l}\text { Range } \\
\text { Mean } \pm S D\end{array}$ & $\begin{array}{c}32 \\
80.600\end{array}$ & $\begin{array}{l}- \\
\pm\end{array}$ & $\begin{array}{c}112 \\
27.981\end{array}$ & $\begin{array}{c}40 \\
91.222\end{array}$ & $\begin{array}{l}- \\
\pm\end{array}$ & $\begin{array}{c}169 \\
52.891\end{array}$ & $\begin{array}{c}60 \\
118.810\end{array}$ & $\begin{array}{l}- \\
\pm\end{array}$ & $\begin{array}{c}219 \\
34.698\end{array}$ & 3.981 & $0.027^{\star}$ & 0.817 & $0.034^{*}$ & 0.177 \\
\hline \multicolumn{2}{|c|}{$\begin{array}{l}\text { Paired } \\
\text { Differences }\end{array}$} & -0.800 & \pm & 3.393 & -16.111 & \pm & 16.174 & -9.000 & \pm & 12.724 & & & & & \\
\hline \multicolumn{2}{|c|}{$\begin{array}{l}\text { Paired Samples } \\
\text { Test }\end{array}$} & \multicolumn{3}{|c|}{0.475} & \multicolumn{3}{|c|}{$0.017^{*}$} & \multicolumn{3}{|c|}{$0.004^{*}$} & & & & & \\
\hline
\end{tabular}

Table 6 Comparison between the control and rehabilitation patients regarding Heart rate recovery (HRR) pre and post rehabilitation

\begin{tabular}{|c|c|c|c|c|c|c|c|c|c|c|c|c|c|c|c|}
\hline \multirow{3}{*}{$\overline{P r e}$} & \multirow{3}{*}{$\begin{array}{l}\text { HRR } \\
\text { Range } \\
\text { Mean } \pm \text { SD }\end{array}$} & \multicolumn{9}{|c|}{ Groups } & \multicolumn{2}{|c|}{ ANOVA } & \multicolumn{3}{|c|}{ TUKEY'S Test } \\
\hline & & \multicolumn{3}{|c|}{ Control } & \multicolumn{3}{|c|}{ Group I } & \multicolumn{3}{|c|}{ Group II } & \multirow{2}{*}{$\frac{F}{9.946}$} & \multirow{2}{*}{$\begin{array}{c}\text { P-value } \\
0.001^{*}\end{array}$} & \multirow{2}{*}{$\begin{array}{c}\text { C\&I } \\
0.645\end{array}$} & \multirow{2}{*}{$\frac{\text { C\&II }}{0.010^{*}}$} & \multirow{2}{*}{$\frac{|\&| \mid}{0.001^{*}}$} \\
\hline & & $\begin{array}{c}2 \\
10.200\end{array}$ & $\begin{array}{l}- \\
\pm\end{array}$ & $\begin{array}{c}17 \\
5.554\end{array}$ & $\begin{array}{c}2 \\
8.333\end{array}$ & $\begin{array}{l}- \\
\pm\end{array}$ & $\begin{array}{c}16 \\
4.330\end{array}$ & $\begin{array}{c}2 \\
15.571\end{array}$ & $\begin{array}{l}- \\
\pm\end{array}$ & $\begin{array}{c}20 \\
4.057\end{array}$ & & & & & \\
\hline Post & $\begin{array}{l}\text { Range } \\
\text { Mean } \pm S D\end{array}$ & $\begin{array}{c}3 \\
10.500\end{array}$ & $\begin{array}{l}- \\
\pm\end{array}$ & $\begin{array}{c}16 \\
4.249\end{array}$ & $\begin{array}{c}4 \\
9.444\end{array}$ & $\begin{array}{l}- \\
\pm\end{array}$ & $\begin{array}{c}13 \\
3.283\end{array}$ & $\begin{array}{c}4 \\
16.429\end{array}$ & $\begin{array}{l}- \\
\pm\end{array}$ & $\begin{array}{c}20 \\
4.285\end{array}$ & 12.542 & $<0.001^{*}$ & 0.840 & $0.002^{*}$ & $<0.001^{*}$ \\
\hline \multicolumn{2}{|c|}{$\begin{array}{l}\text { Paired } \\
\text { Differences }\end{array}$} & -0.300 & \pm & 2.003 & -1.111 & \pm & 2.667 & -0.857 & \pm & 1.621 & & & & & \\
\hline \multicolumn{2}{|c|}{$\begin{array}{l}\text { Paired Samples } \\
\text { Test }\end{array}$} & \multicolumn{3}{|c|}{0.647} & \multicolumn{3}{|c|}{0.247} & \multicolumn{3}{|c|}{$0.025^{*}$} & & & & & \\
\hline
\end{tabular}

Table 7 Comparison between the control and rehabilitation patients regarding Minnesota Living With Heart Failure Questionnaire (MLHFQ) pre and post rehabilitation

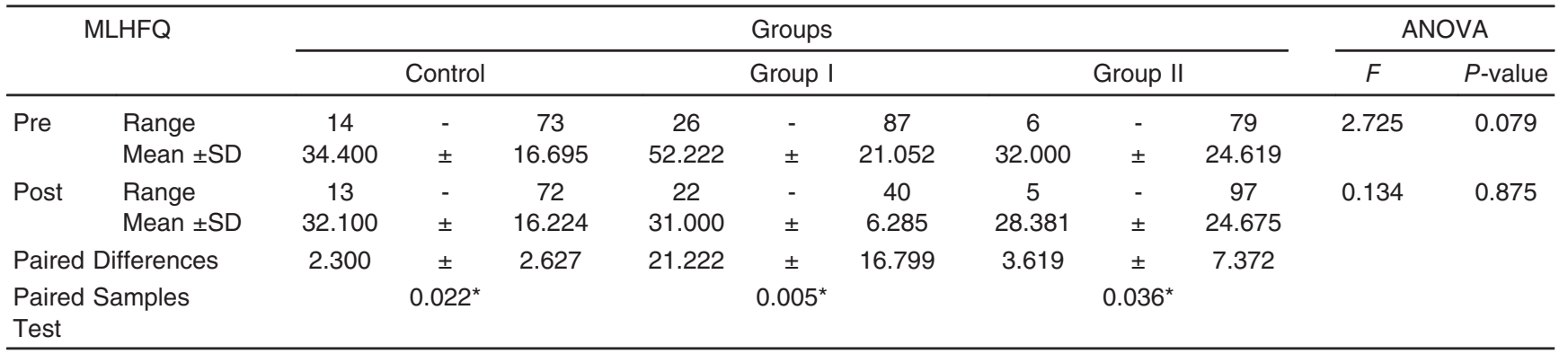

quantitative indicator of exertional hyperapnea, which appears to be a prominent prognostic marker, has been identified previously as able to accurately separate patients by fitness $[29,30]$.

A total of 40 patients diagnosed with chronic stable LHF were selected based on their clinical symptoms and with ejection fraction up to $40 \%$. They were classified randomly into two groups: a control group and a rehabilitation group, which was further divided into group 1 and group 2 according to their functional capacity and prognosis.

Our study showed that both NMES and aerobic training caused a comparable significant improvement of both $\mathrm{VO}_{2} \mathrm{p}$ and $\mathrm{VO}_{2}-\mathrm{VT}$ in both groups 1 and 2 after 
rehabilitation, denoting improved maximum aerobic capacity and endurance. Control group, however, did not show significant improvement regarding both markers. This agrees with previous studies [19,31-38] that stated that both aerobic training and EMS could produce beneficial effects regarding $\mathrm{VO}_{2} \mathrm{p}$ and $\mathrm{VO}_{2}^{-}$ VT. Although EMS produces beneficial effects better than only usual care, such effects are inferior to the aerobic training. This was not the case in our study where the magnitude of change in both EMS and aerobic groups was comparable with no significant difference. This again emphasizes the idea that EMS is more efficient in patients with lower functional capacity. Most patients with very low exercise capacity cannot train at work rates high enough to produce significant improvement owing to dyspnea and extreme fatigueability 19. It also might indicate lower effectiveness when applying EMS to candidates whose functional capacity is already beyond the effective range of EMS; therefore, these patients might not benefit enough from the EMS. On the contrary, any increase, even if modest, in the parameters of functional capacity in patients with low functional capacity would be of great importance enhancing the participants' maximum functional capacity and endurance to perform sustained submaximal activities.

An elevated $\mathrm{VE} / \mathrm{VCO}_{2}$ slope has been linked to poor pulmonary perfusion [39], an impaired cardiac output both at rest and during exercise [40], early lactate accumulation, heightened skeletal muscle, and chemoreceptor sensitivity, and deconditioning [41]. The $\mathrm{VE} / \mathrm{VCO}_{2}$ slope parameter has been recently considered as a very important prognostic factor in patients with $\mathrm{CHF}$ [42]. $\mathrm{VE} / \mathrm{VCO}_{2}$ slope discriminated high-risk and low-risk patients within a $\mathrm{VO}_{2} \mathrm{p}$ range of $10-18 \mathrm{ml} / \mathrm{kg} / \mathrm{min}$ [29]. Patients with $\mathrm{HF}$ with a $\mathrm{VE} / \mathrm{VCO}_{2}$ slope greater than 35 had a mortality rate that was similar to that in patients with a $\mathrm{VO}_{2} \mathrm{P}$ up to $10 \mathrm{ml} / \mathrm{kg} / \mathrm{min}$ [43]. Our study showed that there was a statistically significant improvement of $\mathrm{VE} /$ $\mathrm{VCO}_{2}$ slope in all three groups after the rehabilitation period reflecting better prognosis, with group 1 showing the highest degree of improvement. VE/ $\mathrm{VCO}_{2}$ can be positively affected by aerobic training as well as to improvement in function associated with pharmacological (B-blockade, inhibition of the reninangiotensin-aldosterone axis, sildenafil) interventions $[4,44]$. A previous study [38] showed that there was a significant decrease of $\mathrm{VE} / \mathrm{VCO}_{2}$ slope after either aerobic training, EMS, or combined. The improvement in the EMS group was fully comparable to those of the groups with aerobic training. In our study, improvement was more pronounced in the EMS group. This result might be owing to the fact that our groups were divided according to their functional capacity parameters obtained from the initial CPX, with group 1 having much higher values of $\mathrm{VE} / \mathrm{VCO}_{2}$. On the contrary, group 2 had near-normal values of $\mathrm{VE} / \mathrm{VCO}_{2}$ slope. Thus, improvement was more obvious in the more impaired group.

HRR1 consistently added prognostic value to the VE/ $\mathrm{VCO}_{2}$ slope and other CPX responses [3]. Our study showed that mean HRR1 for group 1 was $8.3 \pm 4.33$ whereas that of group 2 was $15.6 \pm 4.1$. There was a direct significant correlation between $\mathrm{HRR}$ values obtained from the initial CPX and $\mathrm{VO}_{2} \mathrm{p}, \mathrm{VO}_{2}^{-}$ $\mathrm{AT}$, and $\mathrm{WL}$ and inverse correlation with VE/ $\mathrm{VCO}_{2}$ and MLHFQ denoting good correlation with patients' functional capacity and prognostic markers. This comes in accordance with the previous studies $[20,45,46]$ stressing the significance of HRR1 in prediction of adverse events and its relation to other prognostic CPX variables $\left(\mathrm{VO}_{2} \mathrm{p}\right.$ and $\mathrm{VE} / \mathrm{VCO}_{2}$ slope and shorter exercise time).

All the patients in our study were on BBs agents as part of their pharmacological management. According to Racine et al. [47], while improving left ventricular function and prognosis, $\beta$-blocker therapy does not influence HRR.

There was a significant improvement of HRR after rehabilitation in group 2 whereas the difference was insignificant in the control group and group 1 . This was not the case with Stacey et al. [46] who declared that aerobic-based cardiac rehabilitation program had no apparent effect on HRR in patients who have higher initial functional capacities and normal HRR responses ( $\geq 12$ beats/min). This could be attributed to the intensity and duration of exercise program. Another study [48] showed that HRR was significantly more rapid in the exercise group after a 2-month aerobic rehabilitation program compared with control (main effect 12.6 vs. 2.6 beats $/ \mathrm{min}$ in the trained and control groups, respectively, $P<0.005)$. Dimopoulos et al. [49] suggested that continuous rather than interval exercise training improves early HRR1, a marker of parasympathetic activity, suggesting a greater contribution to the autonomic nervous system. These conflicting results may be because of type of recovery used or the intensity and duration of training. Our results showed that patients of group 1 did not show significant improvement of HRR after EMS. As to our knowledge, no studies are available that examined the effect of EMS on HRR. This could be explained by the fact 
that almost $90 \%$ of group 1 experienced DM which has a deleterious effect on the autonomic system which in turns affects HRR besides the short duration of the program.

Our study showed a statistically significant difference of workload in both group 1 and group 2 after rehabilitation period, whereas the control group showed no improvement. Although group 2 showed more improvement, still there was no statistically significant difference between the two groups. This agrees with previous studies stating that either aerobic or EMS training has a positive effect on the peak workload $[31,33,38]$.

Comparison of the MLHFQ values in our study before and after the treatment period yielded a statistically significant difference $(P<0.05)$ in all the three groups, with group 1 showing the most reduction. This agrees with previous studies $[37,38,50]$ that observed a significant improvement in the quality-of-life questionnaires after rehabilitation whether aerobic or EMS.

Although previous studies showed that EMS caused inferior improvements to aerobic training, yet in our study, improvements in the functional parameters in the EMS group were fully comparable to the group of aerobic training, denoting that the physiological response to the increase of oxygen demand during contraction caused by EMS is similar to that caused by conventional physical exercise. This proves our hypothesis that EMS could be equally beneficial when applied to selected patients with lower functional capacity. $\mathrm{VO}_{2}-\mathrm{VT}$ and $\mathrm{VE} / \mathrm{VCO}_{2}$ slope were found to be useful parameters in stratifying patients with HF, allowing us for better selection of those who would benefit most from EMS.

As a considerable number of patients with $\mathrm{CHF}$ tend to abandon physical training owing to the discomfort related to dyspnea, NMES is considered a very useful technique to improve the functional capacity. NMES could be proposed when the patient lacks the motivation for regular physical activity or when the comorbidities and incapacities associated with $\mathrm{CHF}$ make conventional training impossible; NMES is an alternative therapy that can limit loss of muscle volume and strength in patients with $\mathrm{CHF}$, taking into consideration, proper selection of the candidates to gain the maximum benefit. Moreover, the total NMES intervention hours are strongly correlated with change in the functional capacity parameters [37].

\section{Financial support and sponsorship}

Nil.

\section{Conflicts of interest}

There are no conflicts of interest.

\section{References}

1 Colucci W, Braunwald E. Pathophysiology of heart failure. Braunwald's heart disease. A textbook of cardiovascular medicine. 7th ed. Elsevier Saunders; 2005. pp. 509-538.

2 Pina IL, Apstein CS, Balady GJ, Belardinelli R, Chaitman BR, Duscha BD, et al. Exercise and heart failure: a statement from the American Heart Association Committee on exercise, rehabilitation, and prevention. Circulation 2003 107:1210-1225.

3 Arena R, Myers J, Abella J, Pinkstaff S, Brubaker P, Kitzman D, et al. Defining the optimal prognostic window for cardiopulmonary exercise testing in patients with heart failure. Circ Heart Fail 2010; 3:405-4113.

4 Arena R, Myers J, Guazzi M. The clinical and research applications of aerobic capacity and ventilatory efficiency in heart failure: an evidencebased review. Heart Fail Rev 2008; 13:245-269.

5 Hunt SA, Abraham WT, Chin MH, Feldman AM, Francis GS, Ganiats TG, et al. ACC/AHA 2005 Guideline Update for the Diagnosis and Management of Chronic Heart Failure in the Adult: a report of the American College of Cardiology/American Heart Association Task Force on Practice Guidelines (Writing Committee to Update the 2001 Guidelines for the Evaluation and Management of Heart Failure): developed in collaboration with the American College of Chest Physicians and the International Society for Heart and Lung Transplantation: endorsed by the Heart Rhythm Society. Circulation 2005; 112:e154-e235.

6 Lindenfeld J, Albert NM, Boehmer JP, Collins SP, Ezekowitz JA, Givertz $\mathrm{MM}$, et al. HFSA 2010 comprehensive heart failure guideline. J Card Fail 2010; 16:e1-e194.

7 Kucio E, Kucio C. Definition. In: Katowice KC, editor Chronic heart failure. Jerzy Kukuczka Academy of Physical Education in Katowice; 2011.

8 Laura MTN, Lawrence PC, Vinícius Z, Maldaner S, Marianne LS, Ross A, et al. Effect of chronic neuromuscular electrical stimulation on primary cardiopulmonary exercise test variables in heart failure patients: a systematic review and meta-analysis. IJC Metab Endocr 2014; 5:28-35.

9 Banerjee P. Electrical muscle stimulation for chronic heart failure: an alternative tool for exercise training? Curr Heart Fail Rep 2010; 7:52-58.

10 Maddocks M, Gao W, Higginson IJ, Wilcock A. Neuromuscular electrical stimulation for muscle weakness in adults with advanced disease. Cochrane Database Syst Rev 2013; 1:CD009419.

11 Dolgin M, Fox AC, Gorlin R, Levin RI. New York Heart Association. Criteria Committee. Nomenclature and criteria for diagnosis of diseases of the heart and great vessels. 9th ed. Boston, MA: Lippincott Williams and Wilkins.

12 Goldman L, Hashimoto B, Cook EF, Loscalzo A. Comparative reproducibility and validity of systems for assessing cardiovascular functional class: advantages of a new specific activity scale. Circulation 1981; 64:1227-1234.

13 Leidy N, Rentz A, Zycynsky T. Evaluating health-related quality of life outcomes in patients with congestive heart failure. Pharmacoeconomics 1999; 15:19-46.

14 Cohen-Solal A, Beauvais F, Tabet JW. Cardiopulmonary exercise testing in chronic heart failure. Cardiovasc Prev Rehabil 2007; XX:99-109.

15 Naughton J, Sevelius G, Balke B. Physiological responses of normal and pathologic subjects to a modified work capacity test. J Sports Med 1963; 31:201-207.

16 Astrand O, Rodahl K. Textbook of work physiology. New York, NY: McGraw-Hill Book Company; 1986.

17 Ingle L, Goode K, Carroll S, Sloan R, Boyes C, Cleland JG, et al. Prognostic value of the $\mathrm{VE} / \mathrm{VCO}_{2}$ slope calculated from different time intervals in patients with suspected heart failure. Int J Cardiol 2007; 118:350-355.

18 Scardovi A, De Maria R, Coletta C, Aspromonte N, Perna S, Cacciatore G, et al. Multiparametric risk stratification in patients with mild to moderate chronic heart failure. J Card Fail 2007; 13:445-451.

19 Deley G, Eicher JC, Verges B, Wolf JE, Casillas JM. Do low-frequency electrical myostimulation and aerobic training similarly improve performance in chronic heart failure patients with different exercise capacities? J Rehabil Med 2008; 40:219-224.

20 Vera K, Thomas PO, Kent RB, Prabin T, Thomas GA, Bruce DJ. Heart rate recovery and prognosis in heart failure patients. Eur J Appl Physiol 2009; 105:37-45.

21 Raphaela VG, Ligia MA, Thais SN, et al. Electrical muscle stimulation improves neurovascular control and exercise tolerance in hospitalized advanced heart failure patients. Circulation 2015; 132:A12286. 
22 Pozehl B, Duncan K, Hertzog M. The effects of exercise training on fatigue and dyspnea in heart failure. Eur J Cardiovasc Nurs 2007; 7:127-132.

23 Smart N, Haluska B, Jeffriess L, Marwick TH. Exercise training in systolic and diastolic dysfunction:Effects on cardiac function, functional capacity, and quality of life. Am Heart J 2007; 153:530-536.

24 Balady GJ, Arena R, Sietsema K, Myers J, Coke L, Fletcher GF, et al. Clinician's guide to cardiopulmonary exercise testing in adults. A scientific statement from the American Heart Association. Circulation 2010; 122:191-225.

25 Fülster S, Tacke M, Sandek A, Ebner N, Tschöpe C, Doehner W, et al. Muscle wasting in patients with chronic heart failure: results from the studies investigating co-morbidities aggravating heart failure (SICA-HF). Eur Heart J 2013; 34:512-519.

26 Van Tassell BW, Arena RA, Toldo S, Mezzaroma E, Azam T, Seropian IM, et al. Enhanced interleukin-1 activity contributes to exercise intolerance in patients with systolic heart failure. PLoS ONE 2012; 7:e33438.

27 McMurray JJ, Adamopoulos S, Anker SD, Auricchio A, Böhm M, Dickstein $\mathrm{K}$, et al. ESC guidelines for the diagnosis and treatment of acute and chronic heart failure 2012: the Task Force for the Diagnosis and Treatment of Acute and Chronic Heart Failure 2012 of the European Society of Cardiology. Developed in collaboration with the Heart Failure Association (HFA) of the ESC. Eur J Heart Fail 2012; 14:803-869.

28 Gitt AK, Wasserman K, Kilkowski C, Kleemann T, Kilkowski A, Bangert M, et al. Exercise anaerobic threshold and ventilatory efficiency identify heartfailure patients for high risk of early death. Circulation 2002; 106:3079-3084.

29 Corra U, Mezzani A, Bosimini E, Scapellato F, Imparato A, Giannuzzi P. Ventilatory response to exercise improves risk stratification in patients with chronic heart failure and intermediate functional capacity. Am Heart J 2002; 143:418-426.

30 Arena R, Myers J, Aslam SS, Varughese EB, Peberdy MA. Peak $\mathrm{VO}_{2}$ and $\mathrm{VE} / \mathrm{VCO}_{2}$ slope in patients with heart failure: a prognostic comparison. Am Heart J 2004; 147:354e60.

31 Nuhr MJ, Pette D, Berger R, Quittan M, Crevenna R, Huelsman M, et al. Beneficial effects of chronic low-frequency stimulation of thigh muscles in patients with advanced chronic heart failure. Eur Heart J 2004; 25:136-143.

32 Deley G, Kervio G, Verges B, Hannequin A, Petitdant MF, Salmi-Belmihoub $\mathrm{S}$, et al. Comparison of low-frequency electrical myostimulation and conventional aerobic exercise training in patients with chronic heart failure. Eur J Cardiovasc Prev Rehabil 2005; 12:226-233.

33 DobsákP, Nováková M, Fiser B, Siegelová J, Balcárková P, Spinarová L, etal. Electrical stimulation of skeletal muscles. An alternative to aerobic exercise training in patients with chronic heart failure? Int Heart J 2006; 47:441-453.

34 Piepoli MF, Corra U, Agostoni PG, Belardinelli R, Cohen-Solal A, Hambrecht R, Vanhees L. Statement on cardiopulmonary exercise testing in chronic heartfailure due to left ventricular dysfunction: recommendations for performance and interpretation. Part II: How to perform cardiopulmonary exercise testing in chronic heart failure. Eur $\mathrm{J}$ Cardiovasc Prev Rehabil 2006; 13:300-311.

35 Myers J, Hadley D, Oswald U, Bruner K, Kottman W, Hsu L, et al. Effects of exercise training on heart rate recovery in patients with chronic heart failure. Am Heart J 2007; 153:1056-1063.
36 Sbruzzi G, Ribeiro RA, Schaan BD, Signori LU, Silva AM, Irigoyen MC, et al. Functional electrical stimulation in the treatment of patients with chronic heart failure: a meta-analysis of randomized controlled trials. Eur $\mathrm{J}$ Cardiovasc Prev Rehabil 2010; 17:254-260.

37 Smart NA, Dieberg G, Giallauria F. Functional electrical stimulation for chronic heart failure: a meta-analysis. Int J Cardiol 2013; 167:80-86.

38 Vladimir S, Petr D, Michal P, Lenka S, Vitovec J, Krejci J, et al. Exercise training combined with electromyostimulation in the rehabilitation of patients with chronic heart failure: a randomized trial. Biomed Pap Med Fac Univ Palacky Olomouc Czech Repub 2014; 158:98-106.

39 Banning AP, Lewis NP, Northridge DB, Hendersen AH. Perfusion/ ventilation mismatch during exercise in chronic heart failure: an investigation of circulatory determinants. Br Heart J 1995; 74:27-33.

40 Reindl I, Wernecke KD, Opitz C, Wensel R, König D, Dengler T, et al. Impaired ventilatory efficiency in chronic heart failure: possible role of pulmonary vasoconstriction. Am Heart J 1998; 136:778-785.

41 Ponikowski P, Francis DP, Piepoli MF, Davies LC, Chua TP, Davos CH, et al. Enhanced ventilatory response to exercise in patients with chronic heart failure and preserved exercise tolerance: marker of abnormal cardiorespiratory reflex control and predictor of poor prognosis. Circulation 2001; 103:967-972.

42 Jaussaud J, Aimable L, Douard $\mathrm{H}$. The time for a new strong functional parameter in heart failure: the $\mathrm{VE} / \mathrm{VCO}_{2}$ slope. Int $\mathrm{J}$ Cardiol 2011; 147:189-190.

43 Ritt LE, Oliveira RB, Myers J, Arena R, Peberdy MA, et al. Patients with heart failure in the 'intermediate range' of peak oxygen uptake: additive value of heart rate recovery and the minute ventilation/carbon dioxide output slope in predicting mortality. J Cardiopulm Rehabil Prev 2012; 32:141-146.

44 Guazzi M, Arena R. The impact of pharmacotherapy on the cardiopulmonary exercise test response in patients with heart failure: a mini review. Curr Vasc Pharmacol 2009; 7:557-569.

45 Arena R, Guazzi M, Myers J, Peberdy MA. Prognostic value of heart rate recovery in patients with heart failure. Am HeartJ 2006; 151:851.e7-851.e13.

46 Stacey DS, Ezra AA, Charles LS. Heart rate recovery in heart failure patients after a 12-week cardiac rehabilitation program. Am J Cardiol 2006; 97:694-698.

47 Racine N, Blanchet M, Ducharme A, Marquis J, Boucher JM, Juneau M, et al. Decreased heart rate recovery after exercise in patients with congestive heart failure: effect of [beta]-blocker therapy. J Card Fail 2003; 9:296-302.

48 Myers J, Hadley D, Oswald U, Bruner K, Kottman W, Hsu L, et al. Effects of exercise training on heart rate recovery in patients with chronic heart failure. Am Heart J 2007; 153:1056-1063.

49 Dimopoulos S, Anastasiou-Nana M, Sakellariou D, Drakos S, Kapsimalakou S, Maroulids G, et al. Effects of exercise rehabilitation program on heart rate recovery in patients with chronic heart failure. Eur J Cardiovasc Prev Rehabil 2006; 13:67-73.

50 Karavidas A, Parissis J, Arapi S, Farmakis D, Korres D, Nikolaou M, et al. Effects of functional electrical stimulation on quality of life and emotional stress in patients with chronic heart failure secondary to ischaemic or idiopathic dilated cardiomyopathy: a randomised, placebo controlled trial. Eur J Heart Fail 2008; 10:709-713. 